\title{
Estimate of the correlation signal between cosmic rays and BL Lacs in future data
}

\author{
D.S. Gorbunov, ${ }^{1}$ P.G. Tinyakov, ${ }^{1,2}$ I.I. Tkachev, ${ }^{1,3}$ and S.V. Troitsky ${ }^{1}$ \\ ${ }^{1}$ Institute for Nuclear Research of the Russian Academy of Sciences, \\ 60th October Anniversary prospect 7a, 117312 Moscow, Russia \\ ${ }^{2}$ Service de Physique Théorique, Université Libre de Bruxelles, \\ CP225, blv. du Triomphe, B-1050 Bruxelles, Belgium \\ ${ }^{3}$ CERN Theory Division, CH-1211 Geneva 23, Switzerland
}

\begin{abstract}
The existing correlation between BL Lacertae objects (BL Lacs) and cosmic-ray events observed by HiRes experiment provide sufficient information to formulate quantitatively the hypothesis about the flux of neutral cosmic-ray particles originated from BL Lacs. We determine the potential of future cosmic ray experiments to test this hypothesis by predicting the number of coincidences between arrival directions of cosmic rays and positions of BL Lacs on the celestial sphere, which should be observed in the future datasets. We find that the early Pierre Auger data will not have enough events to address this question. On the contrary, the final Pierre Auger data and the early Telescope Array data will be sufficient to fully test this hypothesis. If confirmed, it would imply the existence of highest-energy neutral particles coming from cosmological distances.
\end{abstract}

PACS numbers: 98.70.Sa, 98.54.Cm

\section{INTRODUCTION}

The origin of particles with energies exceeding $10^{19} \mathrm{eV}$ (hereafter ultra-high-energy cosmic rays, or UHECRs) is one of the most intriguing questions of astroparticle physics (for a review, see e.g. Ref. [1]). Within conventional-physics explanations the existence of these particles is attributed to acceleration in yet unknown astrophysical sites. Various candidates have been proposed (see e.g. Ref. [2], for a recent review). Poor (in astronomical standards) angular resolution of the cosmic-ray experiments makes it impossible to identify actual sources directly. Instead, one has to rely on statistical methods.

A clean and indisputable way to perform a statistical analysis is to formulate a hypothesis about the sources before the data are released. This was not possible to do with the first data sets. At present, however, there exists enough data to specify the class of likely sources. More important, new high-quality data are expected from cosmic-ray experiments in the very near future. Before these data are released, a hypothesis to be tested should be formulated and the procedure to verify or falsify it should be defined. In particular, it is important to define quantitatively which results of a particular experiment would test the hypothesis at a given confidence level. In this note we determine such a quantitative procedure.

During the last ten years, many classes of astrophysical objects were tested for positional correlations with arrival directions of UHECRs (see Ref. [3] for a comparative study). Significant correlations were found [4-6] with different samples of BL Lacs from the Veron catalog [7]. The correlations with BL Lacs appear in new independent data sets as well. Most recently the correlations between bright confirmed BL Lacs and the arrival directions of events observed by the HiRes stereo experiment, were found [8] and confirmed by an independent method [9]. This case is particularly clean for it involves minimum of assumptions concerning the BL Lac sample and propagation of UHECR particles (the latter are assumed neutral and propagate along straight lines). Thus, it is most suitable for a rigorous test with the future data. The purpose of this paper is to formulate the testing procedure before the data are released.

We specify the hypothesis in Sec. II and formulate the procedure to test it in the following sections. In Sec. III, we describe how to estimate the experiment-independent quantitative parameter involved in the hypothesis on the basis of a single experiment (HiRes stereo in our case). In Sec. IV, we estimate the sensitivity of future experiments to the suggested hypothesis and determine which results would allow either to exclude or to confirm it at a given confidence level. We calculate the corresponding parameters for the final AGASA, new HiRes stereo, Pierre Auger and Telescope Array data sets.

\section{THE HYPOTHESIS TO BE TESTED}

The most precisely determined published arrival directions were provided by HiRes in the stereo mode [10]. They recorded 271 events with energies $E>10^{19} \mathrm{eV}$, which were found $[8]$ to correlate with bright (visual magnitude $m<18$ ) confirmed BL Lacs from the 10th edition of the Véron catalog [7] — the sample previously identified [5] as correlated with cosmic rays detected by the AGASA and Yakutsk experiments. With the angular resolution (in the stereo mode) of $0.6^{\circ}$, the strongest correlation signal was expected at $0.8^{\circ}$ as determined by Monte-Carlo simulations in Ref. [8]. There were 11 pairs "BL Lac - cosmic ray" found with the separation less than $0.8^{\circ}$ while in average 3.5 pairs were expected for a randomly distributed sample. The angular separations observed are much smaller than a typical deflection of a charged particle of that energy in the Galactic magnetic field, so the correlation may only be explained by the 
presence of a fraction of neutral primary particles [8].

One can summarize these results in the form of a hypothesis suitable for testing by other experiments. The hypothesis consists of three assumptions:

i) Some fraction of cosmic-ray events with energy $E>$ $10^{19} \mathrm{eV}$ corresponds to neutral primary particles.

ii) A part of these neutral particles, or their progenitors, are emitted by the confirmed BL Lacs with magnitude $m<18$ marked "BL" in the catalog [7]. There are 156 such objects. The neutral particles from BL Lacs compose the fraction $\eta$ of the observed UHECR flux.

iii) The observed cosmic-ray fluxes of these 156 sources are roughly equal.

Several remarks concerning the assumptions i)-iii) are in order. Firstly, we determine the value of $\eta$ from the HiRes data. Secondly, the energy threshold of $E=$ $10^{19} \mathrm{eV}$ adopted in i) was used in Ref. [8] because only the arrival directions of the events with $E \geq 10^{19} \mathrm{eV}$ were published in Ref. [10]. Further studies [9] demonstrated the presence of the correlations in an independent (unpublished) data set with $E<10^{19} \mathrm{eV}$. This represents however a different claim which we do not discuss here; it can be analyzed in a similar way. When considering other experiments in what follows we assume the same cut on energy. This assumption may be important for the validity of the predictions, as the fraction of neutral particles (which exhibit the neutral correlations) may vary with energy. If these variations are strong, the energy dependence of the acceptance of the experiments has to be taken into account even at the same energy cut. Also, it is necessary that absolute energy calibrations of different experiments match each other. Otherwise this should be taken into account when selecting events for testing the hypothesis (it is worth noting that, currently, the spectra obtained by different experiments do not match).

Thirdly, the assumption ii) does not exclude other possible sources of UHECRs and means only that BL Lacs emit some of the cosmic-ray particles. We will see shortly that according to HiRes data the fraction $\eta$ of neutral UHECRs attributed to BL Lacs is actually small, of order $2 \%$.

Finally, the assumption (iii) is a technical one; it may be replaced by any other particular conjecture on the relative cosmic-ray brightness of BL Lacs in the sample. In fact, our analysis requires only that in the flux of cosmic rays observed by a given experiment the fraction of events which are associated with BL Lacs (this fraction equals $\eta$ if the experiment sees the whole sky with the uniform acceptance) is proportional to the number of BL Lacs covered by the acceptance area.

Our further logic is as follows. From each cosmic-ray experiment, one can put bounds on the fraction $\eta$ under the assumptions (i)-(iii). Two experiments could, in principle, put the bounds which are, with some probability $p$, inconsistent with each other. Leaving aside the possibility that one of the experiments is wrong, this would mean the rejection of the set of assumptions at the confidence level $p$.

\section{ESTIMATING $\eta$ FROM THE HIRES DATA}

Consider a given catalog of $M$ astrophysical objects (candidate sources) with celestial coordinates $\left\{\alpha_{i}, \delta_{i}\right\}$, $i=1, \ldots, M$, and a cosmic-ray experiment. Depending on the geographical location, field of view and observation time this experiment has different exposures to particular regions of the sky. This can be parametrized by the direction-dependent differential exposure $d A(\alpha, \delta)$, which we normalize to one,

$$
\int d A(\alpha, \delta)=1
$$

The integration here runs over the whole celestial sphere.

Under certain assumptions[17], the number $n$ of observed pairs "source - cosmic ray" separated by angular distances smaller than some (small) angle $\theta$ is described by the Poisson distribution,

$$
P_{n}[S, B]=\frac{(S+B)^{n}}{n !} \cdot \mathrm{e}^{-(S+B)},
$$

where $B$ denotes the average number of background events (that is, either originated from the sources not contained in the catalog or deflected) and $S$ denotes the average number of events which originate from the sources in the catalog (we call $S$ the signal).

The background events are supposed to be isotropically distributed and detected according to the experiment's exposure, hence

$$
B(\theta)=N \sum_{i} \int_{\Omega_{i}} d A
$$

where the sum is taken over all sources in the catalog, $\Omega_{i}$ is a circle of the radius $\theta$ centered on $i$-th source and $N$ is the total number of cosmic-ray events in the data set. This can be written as

$$
B(\theta)=\frac{\pi \theta^{2}}{4 \pi} N M F,
$$

where

$$
F \equiv \frac{4}{M \theta^{2}} \sum_{i} \int_{\Omega_{i}} d A
$$

is a geometrical "correction factor" which describes the distribution of the objects from a given catalog, with respect to the acceptance of the experiment: it equals one for the uniform distribution and is smaller (larger) than one if there is an underdensity (overdensity) of sources within the acceptance area. For small $\theta$ it does not depend on $\theta$. The exact values of $F$ for particular experiments and a particular catalog of sources can be obtained 


\begin{tabular}{|c||c|c|c|}
\hline Experiment & $\sigma$ & Ref. & $F$ \\
\hline HiRes (stereo) & $0.6^{\circ}$ & {$[10,11]$} & 1.38 \\
\hline AGASA & $2.4^{\circ}$ & {$[12]$} & 1.37 \\
PAO (surface) & $1.4^{\circ}$ & {$[13,14]$} & 0.53 \\
PAO (hybrid) & $0.6^{\circ}$ & & 0.48 \\
TA (surface) & $1.55^{\circ}$ & {$[15]$} & 1.41 \\
TA (hybrid) & $0.62^{\circ}$ & & 1.51 \\
\hline
\end{tabular}

TABLE I: Parameters of the cosmic-ray experiments. $\sigma$ is the angular resolution of the experiment (the radius of the circle containing $68 \%$ of the reconstructed events from a point source). Reference is given for the value of $\sigma$ and for the details of direction-dependent exposure. See Eq. (4) for the definition of $F$.

from Eq. (4); for the BL Lac sample under discussion and some ongoing and planned experiments they are listed in Table I.

The signal $S$ is different for different experiments. Let us express it in terms of the fraction $\eta$. For $S \ll N$, the expression is linear[18]. The coefficient of proportionality depends on the acceptance of the experiment, the angular resolution (including the shape of the point-spread function) and on the angle at which the signal is measured. The exact expression reads

$$
S(\theta)=g(\theta) F N \eta
$$

where the factor $g(\theta)$ is the integrated point-spread function of the experiment: this is the fraction of the events observed within the angle $\theta$ from the position of the point source. For instance, for $\theta$ equal to the angular resolution (by definition, the radius of a circle containing $68 \%$ of the events) one has $g\left(\theta_{68}\right)=0.68$ [19].

In a given experiment one measures $n$ and has to determine $\eta$. The likelihood function for $\eta$ can be obtained from Eq. (1) by normalizing $P_{n}$ to $1, \int_{0}^{1} \mathcal{L}(\eta) d \eta=1$. One has

$$
\mathcal{L}(\eta)=\frac{g F N}{\Gamma(n+1, B)}(g F N \eta+B)^{n} \mathrm{e}^{-g F N \eta-B} .
$$

For the original HiRes stereo sample [10], $B=3.5$ and $n=11$ at $\theta=0.8^{\circ}$ [8]. At the $95 \%$ C.L. one has $0.015<$ $\eta<0.035$. Here and in the estimates of the next section we have set $\theta=\sqrt{2} \sigma$, where $\sigma$ is the angular resolution of the relevant experiment (for the discussion of the optimal choice of $\theta$ see Refs. [4, 8] and Sec. IV).

\section{PREDICTIONS FOR FUTURE EXPERIMENTS}

With two different experiments $\mathrm{X}$ and $\mathrm{Y}$, one obtains two likelihood functions, $\mathcal{L}_{X}(\eta)$ and $\mathcal{L}_{Y}(\eta)$. The two-dimensional probability distribution function corresponding to the observation of $\eta_{1}$ in the experiment $\mathrm{X}$ and $\eta_{2}$ in $\mathrm{Y}$ is simply

$$
\mathcal{L}_{X}\left(\eta_{1}\right) \mathcal{L}_{Y}\left(\eta_{2}\right)
$$

\begin{tabular}{|c||c|c|c|c||c||c|}
\hline Experiment & $\theta=\sqrt{2} \sigma$ & $N$ & $S$ & $B$ & $\Delta n_{95 \%}$ & $N_{0}$ \\
\hline HiRes, original & $0.85^{\circ}$ & 271 & 7.46 & 3.54 & & \\
\hline HiRes (stereo) & $0.85^{\circ}$ & 190 & 5.23 & 2.48 & $1-17$ & 271 \\
AGASA & $3.39^{\circ}$ & 1500 & 43.4 & 310 & $308-417$ & 3870 \\
PAO (surface) & $1.98^{\circ}$ & 500 & 5.50 & 13.5 & $10-31$ & 3517 \\
PAO (surface) & $1.98^{\circ}$ & 8000 & 87.9 & 216 & $239-413$ & \\
PAO (hybrid) & $0.85^{\circ}$ & 150 & 1.86 & 0.68 & $0-7$ & 467 \\
PAO (hybrid) & $0.85^{\circ}$ & 2000 & 24.8 & 9.10 & $15-66$ & \\
TA (surface) & $2.19^{\circ}$ & 500 & 15.0 & 44.4 & $42-83$ & 1560 \\
TA (surface) & $2.19^{\circ}$ & 8000 & 239 & 710 & $785-1235$ & \\
TA (hybrid) & $0.88^{\circ}$ & 150 & 4.40 & 2.28 & $1-15$ & 277 \\
TA (hybrid) & $0.88^{\circ}$ & 2000 & 58.6 & 30.3 & $47-161$ & \\
\hline
\end{tabular}

TABLE II: Predictions (95\% CL) for the number $\Delta n_{95 \%}$ of pairs "cosmic ray - BL Lac" separated by less than $\theta$ to be observed by future experiments for the catalog of $156 \mathrm{BL}$ Lac's. The sets of UHECR are constrained by the cuts on energy $\left(E>10^{19} \mathrm{eV}\right)$ and on zenith angle $\left(z<60^{\circ}\right.$ for the Pierre Auger Observatory (PAO) and the Telescope Array (TA), $z<45^{\circ}$ for AGASA). $N$ is the total number of events in a dataset, $S$ and $B$ are the corresponding numbers of average signal and background coincidences. $N_{0}$ is the minimal number of events which the experiment needs to accumulate in order to reach the current HiRes sensitivity.

This formula provides a starting point for quantitative analysis of the compatibility of the two experiments. Changing variables to $\eta_{1}+\eta_{2}$ and $\eta_{1}-\eta_{2}$ and integrating over $\eta_{1}+\eta_{2}$ gives the probability distribution for the difference $\eta_{1}-\eta_{2}$ which tells one how well the two experiments are compatible with each other within our hypothesis. This assumes that the number $n_{Y}$ of pairs "source - cosmic ray" separated by the angle $\theta_{Y}$ observed in the experiment $Y$ is known.

Alternatively, assuming the likelihood function of $\eta$ as follows from the experiment $X$, Eq. (6), one may predict the number of pairs $n_{Y}$ which should be observed by the experiment $Y$ if the hypothesis is correct. The probability distribution for this number is

$$
P_{n_{Y}}=\int_{0}^{1} d \eta \mathcal{L}_{X}\left(\eta, n_{X}\right) \frac{\left(g_{Y} F_{Y} N \eta+B_{Y}\right)^{n_{Y}}}{n_{Y} !} \mathrm{e}^{-g_{Y} F_{Y} N \eta-B_{Y}} .
$$

The $95 \%$ CL interval $\Delta n_{95 \%}=\left(n_{1}, n_{2}\right)$ for the number of pairs to be observed is defined by the conditions

$$
\begin{array}{r}
P\left[n_{1}\right]=0.025, \quad P\left[n_{2}\right]=0.975, \\
\text { where } \quad P[m]=\sum_{n_{Y}=0}^{m} P_{n_{Y}}
\end{array}
$$

(to be more precise, $n_{1}$ is the largest integer for which $P\left[n_{1}\right] \leq 0.025$ and $n_{2}$ is the smallest integer for which $\left.P\left[n_{2}\right] \geq 0.975\right)$.

In Table II we present the expected number of pairs "source - cosmic ray" for several future cosmic-ray data sets. The prediction is done for typical total numbers of 
events $N$ expected to be observed. It is presented in the form of the $95 \%$ CL interval $\Delta n_{95 \%}=\left(n_{1}, n_{2}\right)$. If the number of actually observed pairs falls within the indicated interval, it would be compatible with our hypothesis, otherwise it would exclude it with the probability $95 \%$.

The ability to test the hypothesis is affected by many properties of the experiments. The most important ones are the angular resolution and the total number of events; however, favorable location in the Northern hemisphere (with more known BL Lacs in the field of view) also provides a noticeable advantage. These parameters can be combined into a single quantity - signal-to-noise ratio $Q$ - which characterizes the "sensitivity" of a given experiment to the BL Lac signal. It is defined as the ratio of the signal $S$ to the typical fluctuation of the background,

$$
Q=\frac{S}{\sqrt{B}}=\frac{\sqrt{N F}}{\theta} \cdot \frac{2 g(\theta) \eta}{\sqrt{M}} .
$$

The absolute value of $Q$ is related to the significance at which the existence/absence of the signal can be established. Namely, $Q \gg 1$ is required to test the presence of the signal[20]. More importantly, different experiments can be compared in this way: the one with larger $Q$ is more sensitive to the signal. Assuming that $\theta$ is chosen in the same way for all experiments (for instance, $\theta=\sqrt{2} \sigma$ as in the calculations above), the only experiment-dependent factor in $Q$ is the first factor in Eq. (7). This factor determines the scaling of $Q$ with the total number of events, the optimal angle $\theta$ and the geometrical correction factor $F$. Thus, Eq. (7) allows to estimate the number of events which are necessary for the two experiments to have the same signal-to-noise ratio.

Clearly, in order to test the hypothesis formulated on the basis of the HiRes data, another experiment has to have at least similar sensitivity. It is interesting to see how many events the experiments discussed above have to accumulate to reach the same signal-to-noise ratio as the HiRes experiment. These numbers are presented in Table II. One can see, for instance, that the Pierre Auger experiment will have to collect of order 500 events with $E>10^{19} \mathrm{eV}$ in the hybrid mode (and of order 3500 events observed by the surface detector only) in order to fully probe the hypothesis formulated here.

\section{CONCLUSIONS}

To summarize, in this paper we have formulated, on the basis of the HiRes data, the quantitative hypothe- sis about the connection between UHECRs and BL Lacs (Sect. II, (i)-(iii)) and defined the procedure to test this hypothesis in future experiments. The results are presented in Table II. As follows from these results, the strong correlations observed in the HiRes data are consistent, at the $95 \%$ C.L., even with the absence of signal in some data sets. In particular, the final AGASA data set and the first release of the Pierre Auger data, both expected in near future, may not exhibit any correlations with this sample of BL Lacs and still be consistent with the correlation hypothesis formulated in Sec. II. On the contrary, the absence of correlations in the future Telescope Array and full Pierre Auger data may allow one to falsify this hypothesis. This difference is due to different angular resolutions, exposures and locations of these experiments. Several factors (e.g., the choice of the optimal angle $\theta$ with account of the shape of the individual point-spread function) may improve the sensitivity of the experiments compared to the indicative estimates given in Table II.

In principle, other claims of correlations between UHECR and BL Lacs [4-6] can be tested quantitatively in a similar way. However, due to the cut adjustment (compensated by the penalty factor) to the best signal in these cases the simple analytic relation which in fact determines the flux of cosmic rays produced by BL Lacs, Eq. (6), is no longer valid; its analog may be calculated numerically. We leave this question for future work.

The correlations of BL Lacs with the HiRes stereo data, if confirmed, would imply that a few-percent fraction of the highest-energy cosmic-ray flux is composed of neutral particles coming from cosmological distances. This new UHECR puzzle would be as difficult to solve within conventional physics as the celebrated GZK cutoff [16] problem.

We are indebted to K. Belov, M. Giller and D. Semikoz for helpful and stimulating discussions. This work was supported in part by the INTAS grant 03-51-5112 (D.G., P.T. and S.T.), by the grant of the President of the Russian Federation NS-2184.2003.2, by the grants of the Russian Science Support Foundation (D.G. and S.T.) and by the fellowships of the "Dynasty" foundation (awarded by the Scientific board of ICFPM; D.G. and S.T.). The work of P.T. is supported in part by IISN, Belgian Science Policy (under contract IAP V/27).
[1] M. Nagano and A. A. Watson, Rev. Mod. Phys. 72 (2000) 689 .

[2] D. F. Torres and L. A. Anchordoqui, Rept. Prog. Phys. 671663 (2004) [arXiv:astro-ph/0402371].
[3] D. S. Gorbunov and S. V. Troitsky, Astropart. Phys. 23 (2005) 175 [arXiv:astro-ph/0410741].

[4] P. G. Tinyakov and I. I. Tkachev, JETP Lett. 74, 445 (2001) [Pisma Zh. Eksp. Teor. Fiz. 74, 499 (2001)]. 
[5] P. G. Tinyakov and I. I. Tkachev, Astropart. Phys. 18, 165 (2002).

[6] D. S. Gorbunov et al., Astrophys. J. 577 (2002) L93.

[7] M. P. Véron-Cetty and P. Véron, Astron. Astrophys. 374 (2001) 92.

[8] D. S. Gorbunov, P. G. Tinyakov, I. I. Tkachev and S. V. Troitsky, JETP Lett. 80 (2004) 145 [Pisma Zh. Eksp. Teor. Fiz. 80 (2004) 167].

[9] R.U. Abbasi et al. [High Resolution Fly's Eye Collaboration], arXiv:astro-ph/0507120; C. B. Finley and S. Westerhoff [High Resolution Fly's Eye Collaboration], arXiv:astro-ph/0507465.

[10] R. U. Abbasi et al. [The High Resolution Fly's Eye Collaboration (HIRES)], Astrophys. J. Lett. 610, L73 (2004) [arXiv:astro-ph/0404137].

[11] B. T. Stokes, C. C. H. Jui and J. N. Matthews, Astropart. Phys. 21 (2004) 95 [arXiv:astro-ph/0307491]; Springer R W, 2003, "Stereo spectrum of UHECR showers at the HiRes detector", presentation at the 28th ICRC, Tsukuba.

[12] M. Takeda et al., Astrophys. J. 522 (1999) 225 [arXiv:astro-ph/9902239].

[13] C. Bonifazi et al. [Pierre Auger Collaboration], "Angular resolution of the Pierre Auger Observatory", Proc. 29th ICRC (Pune), 2005.

[14] J.-Ch. Hamilton et al. [Pierre Auger Collaboration], "Coverage and large scale anisotropies estimation methods for the Pierre Auger Observatory", Proc. 29th ICRC (Pune), 2005. arXiv:astro-ph/0507517.

[15] S. Kawakami, "TA project No.55: Toward the discovery of the point sources of highest energy CR by ground array", JPS meeting on 28 - 31 March 2003 (at Tohoku Gakuen Univ.)

[16] K. Greisen, Phys. Rev. Lett. 16, 748 (1966); G. T. Zat- sepin and V. A. Kuzmin, JETP Lett. 4, 78 (1966) [Pisma Zh. Eksp. Teor. Fiz. 4, 114 (1966)].

[17] Poisson distribution of the number of observed pairs "source - cosmic ray" results from the statistical independence of such pairs. If there are two close sources in the catalog, they both may fall within the angle $\theta$ from the arrival direction of a cosmic-ray particle. This breaks the above statistical independence and may cause deviations from the Poisson distribution. Thus, the Poisson distribution works when the number of close (as compared to $\theta$ ) pairs of sources within the acceptance area is small. One may modify slightly the counting procedure to give quantity which is distributed exactly according to Poisson distribution. Namely, one may count cosmic-ray events which fall within a given angular distance $\theta$ from any of candidate sources (so the cosmic-ray events which are close to two candidate sources are not counted twice). We do not use this version of the procedure here for the reasons of compatibility with previous results.

[18] In what follows, we will need to integrate over all possible values of $\eta$ up to 1 , where the linear approximation (5) fails. In practice, one may always reexpress the formulae written in terms of $\eta$ through the signal $S$ and integrate over $0 \leq S \leq \infty$

[19] Since at $\eta \sim 1$ the linear approximation (5) fails, final results depend slightly on the shape of the point-spread function (in this paper, we assumed two-dimensional Gaussian distribution.

[20] The value of $Q$ depends strongly on the angle $\theta$. The latter should be chosen in such a way as to maximize the signal significance. In the cases considered in Refs. [4$6,8]$ this optimal angle was $\theta \approx \sqrt{2} \sigma$. 